\title{
How I Treat: Why Patients with Hematological Malignancies should be admitted to an Intensive Care Unit
}

\author{
Amin T Turki* and Tobias Liebregts \\ Department of Bone Marrow Transplantation, University of Duisburg-Essen, Essen, Germany
}

Received: February 09, 2018; Published: February 19, 2018

*Corresponding author: Amin Turki, MD PhD, Department of Bone Marrow Transplantation, West-German Cancer Cente, University Hospital Essen, University of Duisburg-Essen, Hufelandstrasse 55, 45122 Essen, Germany, Email: amin.turki@uk-essen.de

Keywords: Intensive care treatment; ICU; Hematologic malignancies

Abbreviations: ICU: Intensive Care Units; GVHD: Graft-Versus-Host Disease; ECOG: Eastern Cooperative Oncology Group; APACHE: Acute Physiology and Chronic Health Evaluation

\section{Introduction}

Traditionally, cancer patients are considered poorly suitable for treatment in intensive care units (ICU)[1-7]. In numerous reported studies, ICU and hospital mortality rates were highly above average Tremblay, Hyland et al. [8]. For stem cell recipients, data from the 1990s showed mortality rates up to $90 \%$ Epner, White et al. [4]. Therefore, many clinicians remain skeptical regarding the admission of cancer patients to intensive care units. Because of their potentially curable disease and higher overall survival rates, admission barriers to patients with hematologic malignancies have been recently eased, but for stem cell recipients as well as for patients with metastatic cancer admissions remain highly restrictive. Over the last decade, cancer survivorship has been qualitatively and quantitatively improved. This can be explained through better therapies and improved management of supportive care. Patients with hematologic malignancies did in particular profit from enhanced infectious and sepsis management, but with rising age and multi-morbid patient collectives the absolute number of sepsis death cases doubled National Collaborating Centre for.

As a consequence, the need for intensive care measures comes along with therapeutic options. Therapy-associated complications are supposed to be managed up to intensive care. The long-term outcome of patients with hematologic malignancies and solid tumors is defined by their cure or disease control. For stem cell recipients, remission status and the incidence of GvHD remain critical. On the other hand, the short-term survival of these patient collectives is defined by their intensive care admission diagnosis, ECOG, APACHE II and SOFA scores, as well as intensive care complications. This paradigm shift has been proclaimed by several colleagues, most prominently by Azuay, Mozart et al. [1]. Who could show in 2013 that cancer patients survived the ICU at acceptable levels. In this sense their mortality risk very much resembles the one of other intensive care patients that regularly have extensive comorbidities with high mortality, such as NYHA stage IV heart disease or advanced liver cirrhosis van Vliet, Verburg et al. [5].

While risk factors such as ECOG (Eastern Cooperative Oncology Group) and APACHE (Acute Physiology and Chronic Health Evaluation) scores have been confirmed, ICU admission remains complicated in limit constellations involving hematologic patients with residual disease, metastatic cancer or transplanted patients with graft-versus-host disease (GVHD). A well-reflected and established approach to such dilemma is the ICU admission trial. For suitable persons, ICU admission is subjected to re-evaluation after the first 72h Lecuyer, Chevret et al. [5]. Bird, Farquhar-Smith et al. [2]. If this person is still considered suitable for intensive care treatment, therapy is continued. In an adverse case, the therapy may be terminated and a purely palliative care approach is adopted. The ICU trial does not negate palliative care that certain advanced cancer patients necessarily need, but opens options in constellations that are not entirely clear.The approach shifts acceptance of measures among patients, relatives and caregivers. At our center, we regularly adopted this strategy with success.

Furthermore, structural change in intensive care units might bring advances in the care for cancer patients. Currently, of the 27.489 German intensive care beds, only 301 are located in Hematology/Oncology departments Bundesamt. The creation of specialized intensive care units as well as high case volume has been associated with improved survival Zuber, Tran et al. [10]. As therapies are increasingly concentrated into lager cancer 
centers, the professional management of treatment-associated complications is likely to be further developed. With changing therapeutic possibilities and enhanced survival rates the intensive care management of cancer patients needs to be continuously evaluated. It has been clarified that cancer patients, especially with hematologic malignancies have an equal profit from ICU as other risk collectives. In situations of doubt the $72 \mathrm{~h}$ ICU trial offers a reasonable alternative. Again, because of a rapidly changing therapeutic landscape, new large and prospective clinical trials should further adopt our ICU treatment strategies to this new age in cancer treatment.

\section{References}

1. Azuay E, Mokart D, Pène F, Lambert J, Kouatchet A, et al. (2013) Outcomes of critically ill patients with hematologic malignancies: prospective multicenter data from France and Belgium-a groupe de recherche respirator en reanimation onco-hematologique study. J Clin Oncol 31(22): 2810-2818.

2. Bird GT, Farquhar-Smith P, Wigmore T, Potter M, Gruber PC (2012) Outcomes and prognostic factors in patients with haematological malignancy admitted to a specialist cancer intensive care unit: a 5 yr study. Br J Anaesth 108(3): 452-459.

3. Bundesamt S (2015) Grunddaten der Krankenhäuser. Fachserie, Wiesbaden, Germany, 12: pp. 1-154.

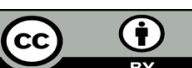

This work is licensed under Creative Commons Attribution 4.0 License

Submission Link: http://biomedres.us/submit-manuscript.php
4. Epner DE, White P, Krasnoff M, Khanduja S, Kimball KT, et al. (1996) Outcome of mechanical ventilation for adults with hematologic malignancy. J Investig Med 44(5): 254-260.

5. Lecuyer L, Verburg S, Thiery G, Darmon M, Schlemmer B, et al. (2007) The ICU trial: a new admission policy for cancer patients requiring mechanical ventilation. Crit Care Med 35(3): 808-814.

6. (2012) National Collaborating Centre for, C National Institute for Health and Care Excellence: Guidance. Neutropenic Sepsis: Prevention and Management of Neutropenic Sepsis in Cancer Patients. London, National Institute for Health and Clinical Excellence (UK).

7. (2012) Copyright (c) National Collaborating Centre for Cancer.

8. Tremblay LN, Hyland RH, Schouten BD, Hanly PJ (1995) Survival of acute myelogenous leukaemia patients requiring intubation/ventilator support. Clan Invest Med 18(1): 19-24.

9. Van Viet M, Overberg IW, van den Boogaard M, de Keizer NF, Peek N, et al. (2014) Trends in admission prevalence, illness severity and survival of haematological patients treated in Dutch intensive care units. Intensive Care Med 40(9): 1275-1284.

10.Zuber B, Tran TC, Aegerter P, Grimaldi D, Charpentier J, et al. (2012) Impact of case volume on survival of septic shock in patients with malignancies. Crit Care Med 40(1): 55-62.

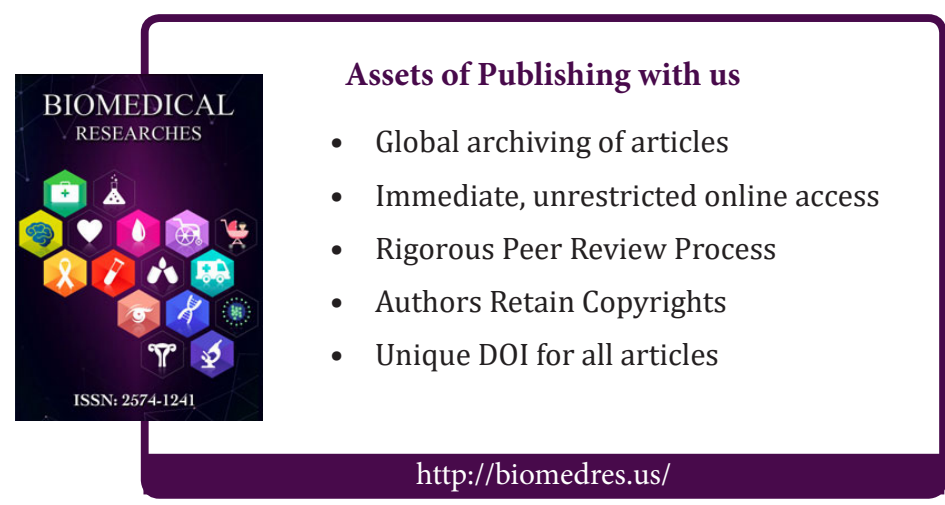

